\title{
Mittelschwere bis schwere Acne vulgaris im Gesicht
}

\begin{abstract}
Das Interesse an einer Akne-Behandlung mit laser- und lichtbasierten Therapien wie der photodynamischen Therapie (PDT), lichtemittierenden Dioden (LED) und "intense pulsed light" (IPL) nimmt weiter zu. Dermatologen aus Kanada und Griechenland haben die Wirkung von blauem Licht in einer Studie mit 90 Patienten untersucht.
\end{abstract}

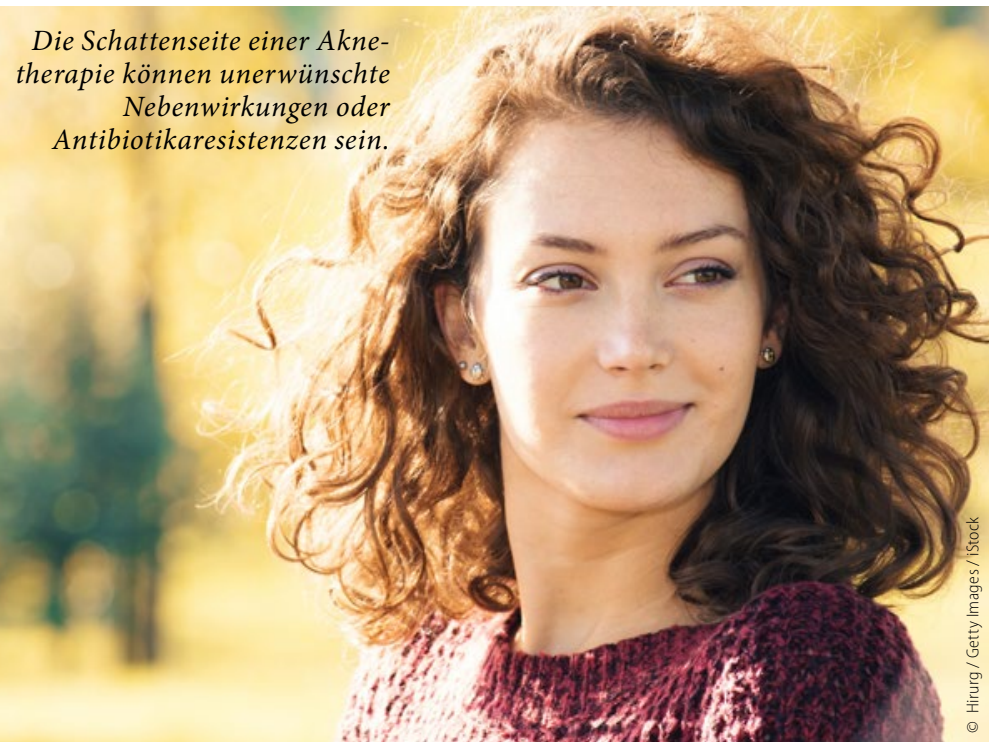

Der Ansatz, sichtbares Licht in der Akne-Therapie zu verwenden, gründet in der Erkenntnis, dass Porphyrin, welches durch Propionibacterium acnes produziert wird, durch Licht angeregt werden kann und somit reaktionsfreudig wird. In der Folge entsteht zytotoxisch reaktiver Sauerstoff, der die Bakterien über eine Zerstörung der Bakterienmembran abtötet. Zugleich findet ein Effekt über die fotodermale Erhitzung der Talgdrüsen statt, erläutern die Studienautoren um Dr. Anreas Nikolis, Québec, Kanada, das Prinzip. Als Vorteile einer Lichttherapie bei Akne nennen die Autoren unter anderem ihren nicht invasiven Charakter sowie die Vermeidung unerwünschter systemischer $\mathrm{Ne}$ benwirkungen. Des Weiteren fände keine Zunahme der Antibiotikaresistenz statt. Die erneute Durchführung im Falle eines Rezidivs stelle kein Problem dar. Ernsthafte topische Nebenwirkungen wie bspw. bei einer PDT, werden ebenfalls nicht beobachtet.

In einer 12-wöchigen, prospektiven, randomisierten Multicenterstudie haben die Dermatologen in Griechenland die Effektivität und Sicherheit einer Chromophor-assistierten Phototherapie mit blauem Licht bei 90 Patienten mit mittelschwerer bis schwerer Acne vulgaris im Gesicht untersucht. Die Patienten waren zwischen 16 und 30 Jahre alt und litten vor Behandlungsbeginn mindestens 6 Monate unter einer aktiven Acne vulgaris. Der überwiegende Teil war weiblich (75,6\%). Da während der Studie die Behandlung nur einer Gesichtshälfte erfolgte (die zweite Hälf- te blieb unbehandelt und diente als Kontrolle), war ein weiteres Einschlusskriterium eine zu Behandlungsbeginn bestehende vergleichbar ausgeprägte Akne in beiden Gesichtshälften, gemessen mithilfe der Investigator's-Global-Assessment(IGA-)Skala.

Auf die zu behandelnde Gesichtshälfte wurde $2 \mathrm{~mm}$ dick ein Fotokonverter-Chromophor-Gel aufgetragen und direkt im Anschluss 5 Minuten mit Multi-LED (415 und $446 \mathrm{~nm}$, LED = light emitting diodes) in einem Abstand von $5 \mathrm{~cm}$ beleuchtet. Die Behandlung wurde 2-mal pro Woche über 6 Wochen hinweg durchgeführt. Alle Patienten verwendeten während der Behandlung und der 6-wöchigen Nachbeobachtung ein Hautreinigungsmittel sowie eine nicht komedogene Creme mit UVSchutz. Als primärer Endpunkt wurde eine Verbesserung um mindestens 2 Punkte auf der IGA-Skala (von 0 bis 4) nach 12 Wochen definiert.

$\mathrm{Zu}$ Beginn der Behandlung zeigten 54 Patienten (60\%) eine mittelschwere (IGA-Grad 3) und 36 Patienten (40\%) eine schwere Akne (IGA-Grad 4). 12 Wochen nach Behandlungsbeginn erreichten 46 Patienten $(51,7 \%)$ mit der behandelten Gesichtshälfte den primären Endpunkt, verglichen mit 16 Patienten (18\%) mit unbehandelter Gesichtshälfte $(\mathrm{p}<0,0001)$. Eine Reduktion von wenigstens $40 \%$ der gezählten Akneläsionen konnte nach 12 Wochen bei 73 Patienten $(81,6 \%)$ in der behandelten Gesichtshälfte festgestellt werden (vs. 41 Patienten [46,0\%] mit unbehandelter Kontrollseite).

\section{Nur geringe, vorübergehende Nebenwirkungen}

In einem Fall trat während der Behandlung ein Pruritus auf, der sich von alleine innerhalb von 5 Minuten wieder legte. Bei 5,6\% der Patienten wurde eine Hyperpigmentierung beobachtet. Bei 6,7\% der Patienten hellte sich das Augenbrauenhaar auf, wenn es bei der Behandlung mit dem Fotokonverter-Gel in Berührung kam. Alle Nebenwirkungen waren vorübergehend und führten in keinem Fall zum Abbruch der Therapie.

Den hohen Anteil an IGA-Verbesserungen in der unbehandelten Gesichtshälfte deuten die Studienautoren einerseits mit Selbstremission, andererseits mit möglichen antiinflammatorischen Effekten durch die Lichttherapie der direkt angrenzenden behandelten Gesichtshälfte. Weiterhin räumen die Autoren zwei Limitierungen ihrer Studie ein, da kein direkter Vergleich mit einer etablierten topischen Aknetherapie erfolgte und ein Großteil der Patienten weiblich war.

Ingo Schroeder

Antoniou C et al. Int J Dermatol 2016; online 30. August 2016; doi:10.1111/ ijd.13349 